\title{
Finite-time Stabilization for Nonholonomic Chained Form Systems with Communication Delay *
}

\author{
Hengjun Zhang, Chaoli Wang ${ }^{\dagger}$ and Gang Wang \\ Department of Control Science and Engineering, \\ University of Shanghai for Science and Technology,Shanghai, 200093, China ${ }^{\ddagger}$ \\ E-mail: clclwang@126.com
}

\begin{abstract}
In this paper, the problem of finite-time stabilization is developed for nonholonomic chained form systems with communication delay in the input. The finite-time control laws are presented by utilizing the switching control strategy and the theory of finite-time stability, which can make the states of the nonholonomic chained form systems to converge from any non-equilibrium state to the equilibrium or a given point in a finite time. Finally, the simulation results show the effectiveness of the proposed control approach.
\end{abstract}

Keywords: Finite-time stabilization, nonholonomic chained systems, communication delay.

* This paper was partially supported by The National Natural Science Foundation (61374040); Key Discipline of Shanghai (S30501); Scientific Innovation program (13ZZ115); Graduate Innovation program of Shanghai (54-13-302-102).

${ }^{\dagger}$ Corresponding author. 


\section{Introduction}

The control and stabilization of nonholonomic systems have received considerable attention during the last few years. Nonholonomic systems with non-holonomic or non-integrable constraints are frequently used to describe the practical control systems. The representative examples of such systems are mobile robots, car-like vehicles, n-level trailer systems and so on. See the survey paper [1] for more details and references therein for more examples. So it is important from the view point of applications to study nonholonomic control systems. From a theorem due to Brockett [2], the nonholonomic system cannot be stabilized at an equilibrium point by pure smooth (or even continuous) state feedback controller.

Therefore, the stabilization problem of non-holonomic systems is still extremely challenging which has attracted a continuously increasing attention in the control community. To overcome this problem, many methods have been proposed, such as continuous timevarying feedback control laws [3], hybrid feedback control laws [4] and discontinuous feedback control laws [5] and so on. Moreover, the robust stabilization problem has been widely researched by using these valid approaches.

However, most models considered are in ideal cases without delays. From a practical point of view, delays in a control system can create significant obstacles to the stabilization problem and potentially degrade the performance of the closed-loop system. It is well known that guaranteeing the stability of a control system with time delay is a challenging problem [6]. The communication delay in the input is related to processing and connecting time for the packets arriving and which also occur when actuators and controllers are connected by networks. So it is very important to study the delay effect on nonholonomic systems. In [7], the classical set-point control problem is studied for rigid robots with input-output communication delays in the closed-loop system. The consensus problem for multiagent systems with input and communication delays is studied based on the frequency-domain analysis in [8]. And the literature [9] presents a control strategy for stabilization of nonholonomic control systems with strongly nonlinear uncertainties and time delay.

In order to get the better convergence speed and higher steady state precision, many researchers have published literature on the finite-time stabilization. And three kinds of global finite-time feedback stabilization methods have been presented as follow: homogeneous properties, backstepping constructive approach and sliding mode technology. A finite-time controller is proposed in [10] for a class of high-order nonlinear by applying homogeneous properties of systems and backstepping constructive approach. The literature [11] considers the finite-time stability of homogeneous systems and gives Lyapunov stability theory of finitetime. Recently, based on backstepping constructive approach, finite-time stabilization is discussed in [12] for a class uncertain nonlinear systems. And the sliding mode technology is investigated in [13]. The distributed control laws in [14] are designed such that multiple nonholonomic mobile agents can be all in agreement within finite time. In [15], the problem of finite-time stability and stabilization for retarded-type functional differential equations is considered.

In this paper, we introduce a class of chained form nonholonomic systems with communication delay in the input and then study the problem of finite-time stabilization for the concerned nonholonomic systems. A global finite-time stabilization controller is presented by using switching control strategy, and making that the all states converge to a given point or the equilibrium point in a finite time.

The paper is organized as follows. In Section II, we will give a formalization of the problem considered in this article and some preliminary lemmas. Section III states our main results including controller design and stability analysis. In Section IV, the simulation results carried out to validate the theoretical framework. Finally, the conclusions of the paper are given in Section V.

\section{Problem Statement}

A class of chained form nonholonomic systems with communication delay in the input to be considered in this paper is described by 


$$
\left\{\begin{array}{l}
\dot{x}_{0}=u_{1}\left(t-\tau_{1}\right), \\
\dot{x}_{1}=x_{2} u_{1}\left(t-\tau_{1}\right), \\
\quad \vdots \\
\dot{x}_{n-1}=x_{n} u_{1}\left(t-\tau_{1}\right), \\
\dot{x}_{n}=u_{2}\left(t-\tau_{2}\right),
\end{array}\right.
$$

where $x=\left(x_{1}, \cdots, x_{n}\right)^{T} \in R^{n}, u_{i}(i=1,2)$ are the inputs of systems, and $u_{i}=u_{i}\left(t-\tau_{i}\right)$ with both $\tau_{1}$ and $\tau_{2}$ being positive constants.

For convenience, some symbols are given in the following at first:

$$
\operatorname{sig}^{\alpha}(x)=|x|^{\alpha} \operatorname{sign}(x),
$$

where $\alpha>0,|x|$ denotes the absolute value of real number $x$ and $\operatorname{sign}(\cdot)$ denotes a sign function.

Next, we will introduce the following lemmas that are needed for our controllers later.

Lemma $2.1^{[11]}$ Consider the following nonlinear system

$$
\dot{y}(t)=f(y(t))
$$

where define that $V: \mathrm{D} \rightarrow R^{n}$ is continuous on an open neighborhood of origin and $f(0)=0$. The equilibrium $x=0$ of the systemis finite-time stable if:

(i) it is asymptotically stable, in $\mathrm{N}$, an open neighborhood of origin, with $\mathrm{N} \subseteq \mathrm{D}$;

(ii) it is finite-time convergent in $\mathrm{N}$, that is, for any initial condition $x_{0} \in \mathrm{N} \backslash\{0\}$, there is a settling time $T>0$ such that every solution $x\left(t, x_{0}\right)$ of system (2) is defined with $x\left(t, x_{0}\right) \in \mathrm{N} \backslash\{0\}$ for $t \in[0, T)$ and satisfies $\lim _{x \rightarrow T} x\left(t, x_{0}\right)=0$, and $x\left(t, x_{0}\right)=0$, if $t \geq T$.

Moreover, if $\mathrm{D}=\mathrm{N}=R^{n}$, the origin is globally finite-time stable equilibrium.

We consider the system

$$
\dot{x}(t)=A x(t)+\sum_{i=0}^{k} B_{i} u\left(t-\tau_{i}\right), \quad t \geq 0,
$$

where $x \in R^{n}, u \in R^{m}, A \in R^{n \times n}, B_{i} \in R^{n \times m}$ and $\tau_{i}$ are positive constants. According to the paper [15], we know that if let

$$
y(t)=x(t)+\sum_{i=0}^{k} L_{\left(A, B_{i}\right)}^{\tau_{i}} u_{t},
$$

where $u_{t}(s)=u(t+s):\left[-\tau_{i}, 0\right] \rightarrow R^{m}$ for every $s \in\left[-\tau_{i}, 0\right]$ and

$$
L_{\left(A, B_{i}\right)}^{\tau_{i}} u_{t}=\int_{-\tau_{i}}^{0} e^{A\left(-\tau_{i}-s\right)} B_{i} u(t+s) d s,
$$

then from (3) and (4), we can have

$$
\dot{y}(t)=A y(t)+B u(t),
$$

with $B=\sum_{i=0}^{k} e^{-A \tau_{i}} B_{i}$.

Lemma 2.2 If the system (5) is finite-time stable by a feedback control law

$$
u(t)=k(t) f(y(t)),
$$

with $k(t)$ bounded and $f: R^{n} \rightarrow R^{n}$ continuous such that $f(0)=0$ and there exists a function $\alpha$ of class $\kappa$ such that $\left\|f(x){ }_{m}\right\| \leq \alpha\left(x_{n}\right)$, then the system (3) is finite-time stable by the feedback controller

$$
u(t)=k(t) f\left(x(t)+\sum_{i=0}^{k} L_{\left(A, B_{i}\right)}^{\tau_{i}} u_{t}\right) .
$$

Now we consider the finite-time stabilization of the integrator systems with delay in the input described by

$$
\left\{\begin{array}{l}
\dot{x}_{1}(t)=x_{2}(t), \\
\quad \vdots \\
\dot{x}_{n-1}(t)=x_{n}(t), \\
\dot{x}_{n}(t)=u(t-\tau),
\end{array}\right.
$$

which can be denoted in short by

$$
\dot{x}(t)=A x(t)+B u(t-\tau)
$$

with

$$
A=\left(\begin{array}{ccccc}
0 & 1 & 0 & \cdots & 0 \\
\vdots & \ddots & \ddots & \ddots & \vdots \\
\vdots & & \ddots & 1 & \vdots \\
0 & \cdots & \cdots & 0 & 0
\end{array}\right), \quad B=\left(\begin{array}{c}
0 \\
\vdots \\
0 \\
1
\end{array}\right) .
$$

According to the Lemma 2.2 and the literature [15], it is not difficult for us to get the following lemma.

Lemma 2.3 Let $k=\left(k_{1}, \cdots, k_{n}\right) \in R^{n}$ such that the polynomial $s^{n}+k_{n} s^{n-1}+\cdots+k_{2} s+k_{1}$ is Hurwitz, 
there exists $\varepsilon \in(0,1)$ such that for all $\alpha \in(1-\varepsilon, 1)$, the system (8) is globally finite-time stable under the continuous feedback control law

$$
u_{\alpha}=-k_{1} \operatorname{sig}^{\alpha_{1}}\left(y_{1}\right)-\cdots-k_{n} \operatorname{sig}^{\alpha_{n}}\left(y_{n}\right),
$$

where

$$
y(t)=x(t)+L_{(A, \bar{B})}^{\tau} u_{t},
$$

and $\bar{B}=e^{-A \tau} B, \alpha_{1}, \cdots, \alpha_{n}$ satisfy

$$
\left\{\begin{array}{l}
\alpha_{i-1}=\frac{\alpha_{i} \alpha_{i+1}}{2 \alpha_{i+1}-\alpha_{i}}, \quad 2 \leq i \leq n \\
\alpha_{n}=\alpha, \alpha_{n+1}=1
\end{array}\right.
$$

\section{Controller Design}

In this section, the main results will be presented. Based on the switching control strategy, the finite-time controllers $u_{1}$ and $u_{2}$ are designed to make that all states of the systems (1) can converge to the equilibrium point in a finite time. To solve the finite-time stabilization problem, we divide the systems (1) into a first-order subsystem

$$
\dot{x}_{0}=u\left(t-\tau_{1}\right),
$$

and a $n$-order subsystem

$$
\left\{\begin{array}{l}
\dot{x}_{1}=x_{2} u_{1}\left(t-\tau_{1}\right), \\
\quad \vdots \\
\dot{x}_{n-1}=x_{n} u_{1}\left(t-\tau_{1}\right), \\
\dot{x}_{n}=u_{2}\left(t-\tau_{2}\right) .
\end{array}\right.
$$

Theorem 3.1The systems (1) can be finite-time stabilization if let $k=\left(k_{1}, \cdots, k_{n}\right) \in R^{n}$ such that the polynomial $s^{n}+k_{n} s^{n-1}+\cdots+k_{2} s+k_{1}$ is Hurwitz. And choosing the proper parameters $c>0$ and $\alpha_{0}, \alpha_{1}, \cdots, \alpha_{n} \in(0,1)$, then take the following switching control laws:

When $t<T_{1}+\tau_{2}$, we have

$$
\left\{\begin{array}{l}
u_{1}=c, \\
u_{2}=-k_{1} \operatorname{sig}^{\alpha_{1}}\left(y_{1}(t)\right)-\cdots-k_{n} \operatorname{sig}^{\alpha_{n}}\left(y_{n}(t)\right),
\end{array}\right.
$$

$$
y_{i}(t)=c^{i-1} x_{i}(t)+L_{(A, \bar{B})}^{\tau_{2}} C^{n-1} u_{2}, \quad \bar{B}=e^{-A \tau_{2}} B .
$$

with the matrix $A$ and $B$ are same as in the Lemma 2.3.

When $t \geq T_{1}+\tau_{2}$, we also have

$$
\left\{\begin{array}{l}
u_{1}=-k_{0} \operatorname{sig}^{\alpha_{0}}\left(x_{0}\right)\left(1+x_{0}\left(t-\tau_{1}\right)^{2}\right), \\
u_{2}=0 .
\end{array}\right.
$$

Proof: When $t<T_{1}+\tau_{2}$, because of $u_{1}=c$, so the $n$-order subsystem (12) can be written as

$$
\left\{\begin{array}{l}
\dot{x}_{1}=c x_{2}, \\
\quad \vdots \\
\dot{x}_{n-1}=c x_{n}, \\
\dot{x}_{n}=u_{2}\left(t-\tau_{2}\right) .
\end{array}\right.
$$

Consider the following transformation

$$
\left\{\begin{array}{c}
\tilde{x}_{1}=c^{0} x_{1}, \\
\vdots \\
\tilde{x}_{n}=c^{n-1} x_{n}, \\
\tilde{u}_{2}=c^{n-1} u_{2} .
\end{array}\right.
$$

With the help of transformation(16), the subsystem (15) can be expressed as

$$
\left\{\begin{array}{l}
\dot{\tilde{x}}_{1}=\tilde{x}_{2}, \\
\quad \vdots \\
\dot{\tilde{x}}_{n-1}=\tilde{x}_{n}, \\
\dot{\tilde{x}}_{n}=\tilde{u}_{2}\left(t-\tau_{2}\right) .
\end{array}\right.
$$

Based on Lemma 2.3, the control law

$$
\tilde{u}_{2}=-k_{1} \operatorname{sig}^{\alpha_{1}}\left(\tilde{y}_{1}(t)\right)-\cdots-k_{n} \operatorname{sig}^{\alpha_{n}}\left(\tilde{y}_{n}(t)\right) \text {, }
$$

where

$$
\tilde{y}_{i}(t)=\tilde{x}_{i}(t)+L_{(A, \bar{B})}^{\tau_{2}} \tilde{u}_{2}, \quad \bar{B}=e^{-A \tau_{2}} B,
$$

the $A$ and $B$ are the same as in the Lemma 2.3, and $\alpha_{1}, \cdots, \alpha_{n}$ satisfy the conditions (10). It can render the subsystem (17) globally finite-time stable. Substituting the transformation (16) into the control law (18), the states $X_{1}, X_{2}, \cdots, X_{n}$ and $u_{2}$ can converge to the origin in a finite time $T_{1}+\tau_{2}$ via the control law (13).

where 


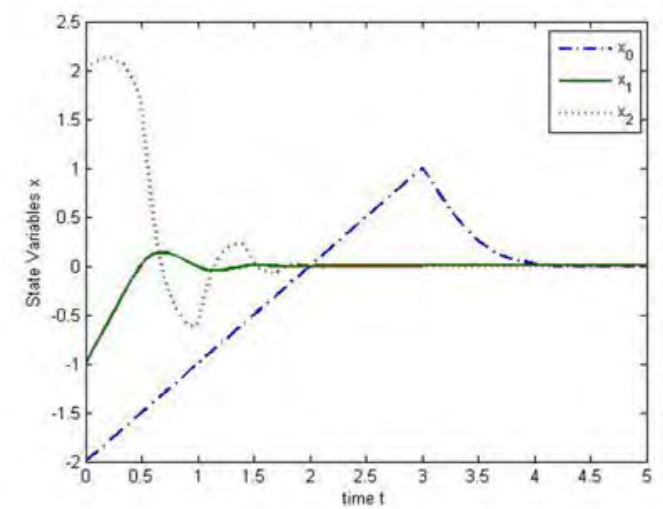

Fig. 1 The state response of system (21)
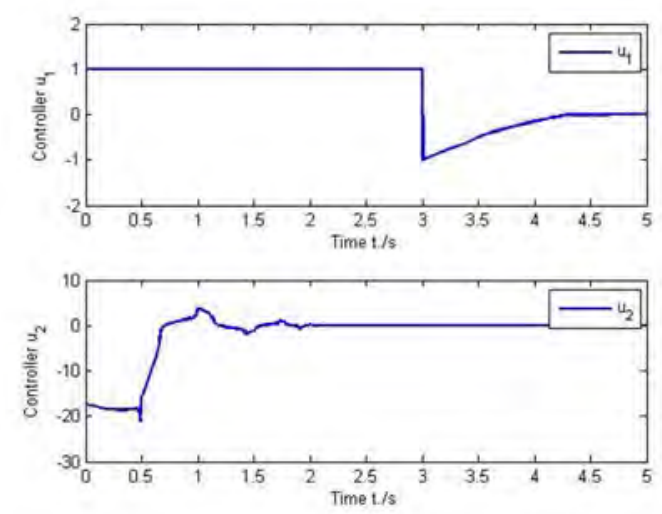

Fig. 2 The response of controllers

When $t \geq T_{1}+\tau_{2}$, the states $x_{1}, x_{2}, \cdots, x_{n}$ have reached on the equilibrium point, thus, we only consider the first order subsystem (11).

Construct a Lyapunov function

$$
V\left(x_{0}(t)\right)=\frac{1}{2} x_{0}(t)^{2} \text {. }
$$

Taking the time derivative of $V\left(x_{0}\right)$ along

$$
\begin{aligned}
\dot{V} & =x_{0} u_{1}=-\left|x_{0}\right|^{1+\alpha_{0}}\left(1+x_{0}\left(t-\tau_{1}\right)^{2}\right) \\
& \leq-\left|x_{0}\right|^{1+\alpha_{0}}=-2^{\left(1+\alpha_{0}\right) / 2} V^{\left(1+\alpha_{0}\right) / 2} .
\end{aligned}
$$

So the subsystem (11) can be stabilized within the settling time $T_{2} \leq\left|x_{0}(t)\right|^{1-\alpha_{0}} /\left(1-\alpha_{0}\right)$ via the switching control law (14). Hence, the statevariables $x_{0}, x_{1}, \cdots, x_{n}$, and the control inputs $u_{1}, u_{2}$ can converge to the equilibrium point after thetime $T=T_{1}+T_{2}+\tau_{1}+\tau_{2}$. This completes the proof of Theorem 3.1.

\section{Simulation}

In this section, we will give an illustrative example of Theorem 3.1. Consider the following nonholonomic chained form systems with communication delay

$$
\left\{\begin{array}{l}
\dot{x}_{0}=u_{1}\left(t-\tau_{1}\right), \\
\dot{x}_{1}=x_{2} u_{1}\left(t-\tau_{1}\right), \\
\dot{x}_{2}=u_{2}\left(t-\tau_{2}\right) .
\end{array}\right.
$$

Choosing $\tau_{1}=\tau_{2}=0.5$ and the initial values of system (21)are $\left(x_{0}, x_{1}, x_{2}\right)=(-2,-1,2)$. Selecting the parameters $k_{1}=2, k_{2}=3, c=1$ and $\alpha_{0}=0.5$, $\alpha_{2}=0.4$, by the formula (10), we have $\alpha_{1}=0.25$.

From Fig. 1, it is easy to see that the state variables of systems can be driven to the equilibrium point in a finite time. As shown in Fig. 2, the control inputs $u_{1}$ and $u_{2}$ can also converge to the origin.

\section{Conclusions}

In this paper, a globally finite-time stabilization problem is presented for nonholonomic chained form systems with communication delays in the input. By utilizing finite-time stability theory and switching control strategy, the nonholonomic chained systems can be divided into different subsystems, and then the controllers of subsystems are given with different methods such that each state of the systems can converge to the equilibrium point in a finite time. Finally, a simulation example is provided to verify that the designed controllers are effective.

\section{References}

1. I. Kolmanovsky, N. H. McClamroch, Developments in nonholonomic control systems. IEEE Contr. Syst. Mag. 15(6)(1995)20-36.

2. R. W. Brockett, Asymptotic stability and feedback stabilization. In Proc. Differential Geometric Control Theory, (Birkhauser, Boston,1983)181-208.

3. Y. P. Tian, S. Li, Exponential stabilization of nonholonomic dynamic systems by smooth time-varying control. Automatica. 38(7)(2002)1139-1146.

4. A. P. Aguiar, A. Pascoal, Stabilization of the extended nonholonomic double integrator via logic based hybrid 
control. In Proc. of 6th international IFAC symposium on robot control, (Vienna Austria, 2000)pp. 208-213.

5. M. C. Laiou, A. Astolfi, Discontinuous control of high order generalized chained systems. Systems Control Letters. 37(1999)309-322.

6. J. P. Richard, Time-delay systems: An overview of some recent advancesand open problems. Automatica. 39(10)(2003)1667-1694.

7. Y. C. Liu, N. Chopra, Control of robotic manipulators under input/output communication delays: theory and experiments. IEEE Transactions on Robotics. 28(3)(2012)742-751.

8. Y. P, Tian, C. L. Liu, Consensus of multi-agent systems with diverse input and communication delays. IEEE Transactions on Automatic Control. 53(9)(2008)21222128.

9. Y. Y. Wu, Y. Q. Wu, Robust stabilization of delayed nonholonomic systems with strong nonlinear drifts. Nonlinear Analysis: Real World Applications. 11(5)(2010)3620-3627.

10. Y. G. Hong, Finite-time stabilization and stabilizability of a class of controllable systems. Systems Control Letters. 46(4)(2001)231-236.

11. S. P. Bhat, D. S. Bernstein, Finite-time stability of homogeneous systems. inProc. of the 1997 American Control Conference. Albuquerque,(New Mexico: American Automatic Control Council,1997), pp. 25132514.

12. X. Q. Huang, W. Lin, B. Yang, Global finite-time stabilization of a class uncertain nonlinear systems. Automatica. 41(5)(2005)881-888.

13. Y. G. Hong, G. W. Yang, D. Z. Cheng, et al., Finite- time convergent control using terminal sliding mode. Journal of Control Theory and Applications. 2(1)(2004)69-74.

14. J. K. Wang, Z. H. Qiu, G. S. Zhang et al., Finite time consensus for multiple non-holonomic agents with communication delay. inProc. of the 31st Chinese Control Conference. (2012),pp. 6267-6272.

15. E. Moulaya, M. Dambrineb, et al., Finite-time stability and stabilization of time-delay systems. Systems Control Letters. 57(7)(2008)561-566. 